\title{
Zoledronic acid inhibits infiltration of tumor-associated macrophages and angiogenesis following transcatheter arterial chemoembolization in rat hepatocellular carcinoma models
}

\author{
DA-YONG ZHOU ${ }^{1 *}$, JIE QIN $^{2 *}$, JIAN HUANG $^{1}$, FENG WANG $^{3}$, GUO-PENG XU ${ }^{4}$, \\ YAN-TIAN LV ${ }^{4}$, JI-BIN ZHANG ${ }^{5}$ and LI-MING SHEN ${ }^{1}$
}

\begin{abstract}
${ }^{1}$ Department of Interventional Radiology and Vascular Surgery, Suzhou Municipal Hospital Affiliated to Nanjing Medical University, Suzhou, Jiangsu 215002; ${ }^{2}$ Department of Anatomy, Histology and Embryology, Shanghai Medical College, Fudan University, Shanghai 200032; Departments of ${ }^{3}$ Pathology, ${ }^{4}$ Respiration and ${ }^{5}$ Radiology, Suzhou Municipal Hospital Affiliated to Nanjing Medical University, Suzhou, Jiangsu 215002, P.R. China
\end{abstract}

Received February 19, 2016; Accepted February 3, 2017

DOI: $10.3892 / \mathrm{ol} .2017 .6717$

\begin{abstract}
Hepatic transcatheter arterial chemoembolization (TACE), a minimally invasive procedure to block the blood supply of tumors and release of cytotoxic agents, is preferentially applied to patients with hepatocellular carcinoma (HCC) who are not able to receive radical treatments. However, the long-term effects of TACE are unsatisfactory, as the microenvironment following procedure stimulates tumor angiogenesis, which promotes recurrence and metastasis of residual tumors. Tumor associated macrophages (TAMs) have been revealed to stimulate tumor growth and angiogenesis associated with poor prognosis in HCC. The present study focused on the changes in TAMs following TACE, and explored the effects of TACE in combination with the TAM inhibitor zoledronic acid (ZA) in rat HCC models. Orthotropic HCC rats were divided into three groups: Sham TACE, TACE alone and TACE combined with ZA treatment. At 7 or 14 days following TACE, tumor growth was evaluated by magnetic resonance imaging (MRI). Infiltration of TAMs was assessed by histological analysis and flow cytometry. Tumor angiogenesis was measured as the mean vessel density, and initial slope was calculated from dynamic contrast enhancement MRI. Local and systemic levels of vascular endothelial growth factor (VEGF) were determined by western blotting or an ELISA,
\end{abstract}

Correspondence to: Dr Da-Yong Zhou, Department of Interventional Radiology and Vascular Surgery, Suzhou Municipal Hospital Affiliated to Nanjing Medical University, 26 Dao Qian Road, Suzhou, Jiangsu 215002, P.R. China

E-mail: sz_dyzhou@hotmail.com

*Contributed equally

Key words: zoledronic acid, tumor-associated macrophages, angiogenesis, transcatheter arterial chemoembolization, hepatocellular carcinoma respectively. The results revealed that TACE inhibited tumor growth at 7 days following the procedure, but this inhibition was attenuated at 14 days following the procedure compared with the sham TACE control. If combined with ZA treatment, TACE exhibited a stable inhibition effect on tumor growth until the end of observation. Investigation of the underlying mechanisms demonstrated that TACE combined with ZA treatment inhibited infiltration of F4/80 positive TAMs and tumor angiogenesis compared with the TACE alone group at 14 days following the procedure. Additionally, the combination treatment significantly inhibited secretion of VEGF in the present models. In conclusion, ZA treatment enhanced the effects of TACE through inhibiting TAM infiltration and tumor angiogenesis in rat HCC models.

\section{Introduction}

Prognosis of hepatocellular carcinoma (HCC), a hypervascular cancer, remains poor due to early recurrence and metastasis following treatment (1). It has become the second leading cause of cancer-associated mortality worldwide $(2,3)$. Asia accounts for $\sim 80 \%$ of newly developing HCC cases globally (4). The majority of patients with HCC are at the intermediate or advanced stage when diagnosed, and are therefore not amenable to curative resection or transplantation (4). Consequently, hepatic transcatheter arterial chemoembolization (TACE), a minimally invasive procedure to block the blood supply of tumors and release of cytotoxic agents, is recommended to patients who cannot undergo surgical resection or local ablation (5-7). Although TACE prior to curative treatment may induce tumor necrosis in certain patients, the long-term effects of TACE are unsatisfactory and survival benefits from this procedure remain controversial $(8,9)$. TACE induces significant tumor necrosis and severe hypoxia (10-12). Local tissue and plasma exhibit an increased number of angiogenic factors following TACE $(13,14)$. Such a microenvironment tilts the balance toward stimulatory angiogenesis, which accelerates recurrence and metastasis of residual tumors following TACE $(13,14)$. Therefore, therapeutic strategies for inhibiting 
local angiogenesis may improve the outcome of patients with HCC treated with TACE.

Tumor infiltrating cells, including the majority of macrophages, are considered to perform a pivotal role in remodulation of tumor microenvironments (15-17). Clinical and epidemiological evidence has indicated that the occurrence and promotion of HCC are accompanied by these inflammatory cells (15-17). Tumor associated macrophages (TAMs), a primary subset of tumor infiltrating cells, may stimulate tumor growth and angiogenesis as M2 polarization through the expression of cytokines, chemokines, growth factors, and matrix metalloproteases $(18,19)$. A high density of TAMs was associated with more microvessels and poor prognosis in the majority of types of tumors, including HCC (20-24). However, few studies have investigated the behavior of TAMs in HCC following TACE. The present study focused on the changes in TAMs following TACE, and explored the effects of combination with the TAM inhibitor zoledronic acid (ZA) in rat HCC models.

\section{Materials and methods}

Rat HCC models. All Sprague-Dawley rats (male; age, 10-14 weeks; body weight, $230 \pm 15 \mathrm{~g}$ ) were obtained from the Animal Experimental Center of Nanjing Medical University (Nanjing, China). The rats were maintained under specific pathogen-free conditions and were fed with sterilized rodent diet and water ad libitum. A total of $1 \times 10^{6}$ Walker-256 cells (Shanghai Institute of Cell Biology, Chinese Academy of Sciences, Shanghai, China) per rat were injected into rat flanks. Rats were sacrificed when tumors grew to $10 \mathrm{~mm}$ in diameter. Tumors were then harvested for implantation into rat livers. At 12 days following implantation, tumor xenografts grew to $\sim 7 \mathrm{~mm}$ in diameter, and these orthotopic HCC models were used for subsequent studies (25).

A total of 18 rats were randomly divided into 3 groups $(n=6)$ : A Sham TACE group [sham TACE at day 0 with a subsequent daily intraperitoneal injection (i.p.) of PBS]; a TACE alone group (TACE at day 0 with a subsequent daily i.p. of PBS); and a TACE combined with ZA (Norvartis International AG, Basel, Switzerland) treatment group [TACE at day 0 with a subsequent daily i.p. of ZA solution $(100 \mu \mathrm{g} / \mathrm{kg}$ body weight)]. Rats were sacrificed with carbon dioxide asphyxiation (1 liters/min flow rate of $\mathrm{CO}_{2}$ in a 10-liter cage, $20 \%$ of cage volume of $\mathrm{CO}_{2}$ and finally, mortality was ensured by lack of cardiac pulse) at 7-14 days following TACE. Animal studies were approved by the Institutional Animal Care and Use Committee of Nanjing Medical University.

TACE procedure. Through a subxiphoid median incision, gastroduodenal arteries were inserted retrogradely with $2-\mathrm{F}$ silicone tubing with a tip located at hepatic artery. Lipidol $(0.3$ $\mathrm{ml}$; Laboratories Gilbert, Hérouville Saint Clair, France) was then injected into the rat liver while securing the branches of the right lobe arteries. Following ligation of gastroduodenal artery and suture of abdomen, rats were allowed to recover (25).

Immunohistochemical staining. Under light microscope (Olympus Corporation, Tokyo, Japan; magnification, x40), implanted HCC tumors obtained from livers following animal euthanasia were examined. Tumor samples $\left(\sim 5 \times 5 \times 5 \mathrm{~mm}^{3}\right)$ were washed (5 min each time for 3 washes) with pre-cooled PBS and fixed with $4 \%$ paraformaldehyde at $4^{\circ} \mathrm{C}$ for $24 \mathrm{~h}$. Subsequently, samples were embedded into Tissue-Tek O.C.T compound (Sakura Finetek USA, Inc., Torrance, CA, USA) and cut into tissue sections (10- $\mu \mathrm{m}$ thick) for histological analysis. Vessel density of tumor tissues was assessed by immunohistochemical staining with anti-cluster of differentiation (CD) 31 antibody (1:200 dilution; Abcam, Cambridge, UK; cat. no., ab119339). Following overnight incubation with CD31 antibody at $4^{\circ} \mathrm{C}$, sections were incubated with horseradish peroxidase conjugated secondary antibody (1:1,000 dilution; Abcam; cat no., ab6789) for an hour at room temperature. Mean vessel density (MVD) was calculated as previously described (26). Briefly, sections were scanned using a light microscope (Olympus corporation, Tokyo, Japan) at a magnification, $\mathrm{x} 40$ and the region with the highest microvascular density (neovascular hotspot) was identified. This region was counted at a magnification of x200 for microvasculature, highlighted by CD31-positive staining. Using the same method, TAMs in tumor tissues were detected by immunohistochemical staining with anti-F4/80 antibody (1:100 dilution; Abcam; cat. no., ab100790) followed by Cy3-conjugated secondary antibody (1:500 dilution; Abcam; cat no. ab6939).

Flow cytometry analysis. Tumor tissues were harvested under a light microscope (Olympus corporation, Tokyo, Japan; magnification, x40) and digested with collagenase IV solution (1 mg/ml; Sigma-Aldrich, Merck KGaA, Darmstadt, Germany) at $37^{\circ} \mathrm{C}$ for $30 \mathrm{~min}$. Isolated cells were filtered through a nylon mesh and washed with media supplemented with $10 \%$ fetal bovine serum (Gibco; Thermo Fisher Scientific, Inc., Waltham, MA, USA). Subsequently, lymphocytes in tumor tissues were purified using Percoll (Sigma-Aldrich; Merck KGaA) gradient centrifugation $(48,000$ x g, $10 \mathrm{~min}$; at $\left.4^{\circ} \mathrm{C}\right)$. These tumor infiltrating lymphocytes were stained with fluorescence-conjugated anti-F4/80 antibody (1:200 dilution; eBioscience, Inc., San Diego, CA, USA; cat. no., 11-4801) in the dark for $15 \mathrm{~min}$ at room temperatureFlow cytometric analysis was performed on a FACSCalibur flow cytometer (BD Biosciences, Franklin Lakes, NJ, USA). Data was expressed as the percentage of F4/80-positive cells in the tumor infiltrating lymphocyte population.

Measurement by dynamic contrast enhancement-magnetic resonance imaging (DCE-MRI). MRI (Magnetom avanto; Siemens AG, Munich, Germany) and image processing (Syngo MR 2004 Version; Siemens) was used to measure tumor volume and assess intensity-time curves with dynamic contrast enhancement. Gadolinium-pentetic acid (Magnevist, Bayer, Newbury, UK) was applied for enhancement with $0.1 \mathrm{mmol} / \mathrm{kg}$ body weight. T2 weighted images (WIs) were first obtained, and then a series of dynamic enhancements (25 series in total) were collected using multi-section T1-weighted fast spoiled gradient-recalled acquisition. The following parameters were applied: Repetition time/echo time, $9.58 \mathrm{msec} / 3.76 \mathrm{msec}$; flip angle, $25^{\circ}$; matrix, 192x192; section thickness, $3 \mathrm{~mm}$; number of signals acquired, 2 ; and field of view, $75 \times 75 \mathrm{~mm}$. Tumor volume $(\mathrm{V})$ was calculated based on $\mathrm{T} 2 \mathrm{WI}: \mathrm{V}=\left(\begin{array}{lll}\mathrm{a} & \mathrm{x} & \mathrm{b}^{2}\end{array}\right) / 3$ 
(a, long diameter; b, short diameter). Contrast enhancement-time curves were constructed from perfusion image series. Initial slope (IS; slope of the curve at the time point of the maximal contrast agent inflow) was calculated according to the formula IS=max $\left[\mathrm{d}\left(\mathrm{CTC}_{i}\right) / \mathrm{dt}\right] . \mathrm{CTC}_{i}$ (curve at the time point $i)$ was calculated according to the formula $\mathrm{CTC}_{i}=\left(\mathrm{I}_{i}-\mathrm{I}_{o}\right) / \mathrm{I}_{o} . \mathrm{I}_{i}$ is the signal intensity at perfusion imaging at time point $i$, and $\mathrm{I}_{o}$ is the signal intensity at the baseline time point. The time following contrast agent administration is indicated by $\mathrm{t}(27,28)$.

Western blot analysis. Following washing (5 min each time for 3 washes) with cold PBS, tumor tissues were suspended with radioimmunoprecipitation assay lysis buffer (Beyotime Institute of Biotechnology, Shanghai, China; cat. no., P0013) containing proteinase inhibitors. Tissues were lysed completely for $30 \mathrm{~min}$ on ice, and whole-cell protein extracts from them were prepared by centrifugation at $12,000 \mathrm{x} \mathrm{g}$ for $20 \mathrm{~min}$ at $4^{\circ} \mathrm{C}$. Protein concentrations were determined using enhanced BCA Protein Assay Kit (Beyotime Institute of Biotechnology; cat. no. P0010) according to the manufacturer's protocol. Protein extracts ( $20 \mu \mathrm{g}$ protein per lane) were separated by $10 \%$ SDS-PAGE and then transferred to polyvinylidene fluoride membranes (EMD Millipore; Billerica, MA, USA). Membranes were incubated with anti-VEGF primary antibody (1:400 dilution; Abcam; cat. no., ab53465) overnight at $4^{\circ} \mathrm{C}$. Following washing $(5 \mathrm{~min}$ each time for 3 washes) with PBS membranes were incubated with horseradish peroxidase conjugated secondary antibody $(1: 5,000$ dilution; cat. no., ab6721) for $1 \mathrm{~h}$ at room temperature. Bands were detected by an enhanced chemiluminescence system (Pierce ECL Western Blotting kit, Thermo Fisher Scientific, Inc.) The expression level of the proteins were normalized to B-actin.

ELISA. Following euthanasia, rats underwent laparotomy and blood samples were drawn from the inferior vena cave prior to harvest of tumor tissues. Plasma were collected following centrifugation $\left(1,000 \mathrm{x} \mathrm{g}\right.$ for $10 \mathrm{~min}$ at $\left.4^{\circ} \mathrm{C}\right)$, and levels of VEGF in them were determined with use of a quantitative rat VEGF ELISA kit (Abcam; cat. no., ab100786) according to the manufacturer's protocol. All analyses were performed in duplicate.

Statistical analysis. Data was presented as the mean \pm standard deviation. Significance between groups was evaluated using two-tailed Student's t-tests, one-way analysis of variance with the post-hoc test of Student Newman Keuls or Tamhane's T2, or $\chi^{2}$ tests as appropriate, with the use of SPSS 11.0 (SPSS, Inc., Chicago, IL, USA). $\mathrm{P}<0.05$ was considered to indicate a statistically significant difference.

\section{Results}

Combination of TACE with ZA treatment enhances inhibition of TACE on tumor growth in rat HCC models. To reveal the effects of TACE combined with or without ZA treatment in rat HCC models, MRI was used to evaluate tumor growth until 14 days following TACE. As presented in Fig. 1, the TACE alone group had reduced tumor volume compared with the

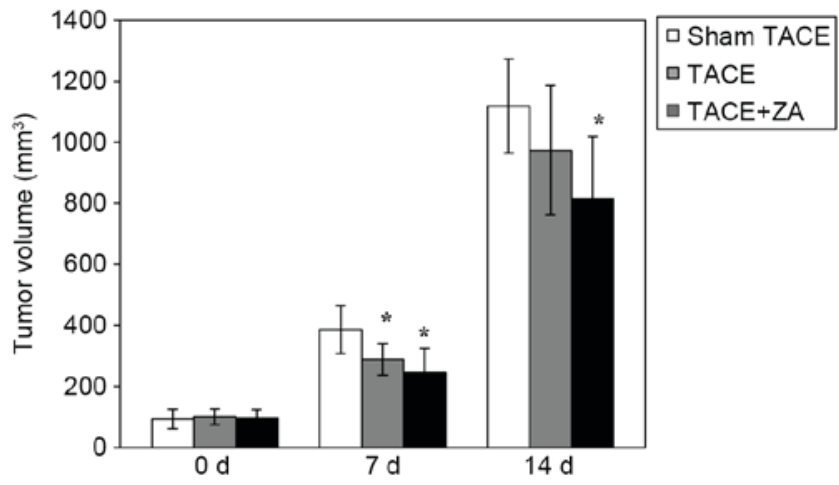

Figure 1. Tumor volume following TACE in rat HCC models. At 0,7 and 14 days following the procedure, orthotopic tumor volumes of $\mathrm{HCC}$ rats from the sham TACE, TACE alone and TACE combined with ZA treatment groups were measured with magnet resonance imaging. The data were expressed as the mean \pm standard deviation of three separate experiments. ${ }^{*} \mathrm{P}<0.05$ vs. sham TACE group. TACE, transcatheter arterial chemoembolization; HCC, hepotocellular carcinoma; ZA, zoledronic acid.

sham TACE group at 7 days subsequent to the procedure $(\mathrm{P}<0.05)$. After 14 days following the procedure, inhibition of tumor growth of TACE alone group was attenuated, yet no significant difference in tumor volume was observed between these two groups (TACE alone vs. sham TACE, P>0.05) If combined with $\mathrm{ZA}$ treatment subsequent to the procedure, TACE displayed a stable inhibitory effect on tumor growth until the end of observation (14 days following TACE; $\mathrm{P}<0.05$ ). These data indicated that combination with ZA treatment enhanced TACE-mediated inhibition of tumor growth in rat HCC models.

ZA inhibits TAM infiltration following TACE. ZA has been demonstrated to be an inhibitor of TAMs in various tumors. Therefore, in the present study, TAM infiltration was firstly examined in the HCC models by immunohistochemical staining. Histological analysis revealed that compared with the sham control group, the TACE alone group had more F4/80-positive TAMs infiltrating orthotopic tumors at 14 days following the procedure. However, TAM infiltration was markedly inhibited in the TACE combined with ZA treatment group (Fig. 2A). In addition, quantitative analysis of isolated tumor infiltrating cells by flow cytometry validated these findings. The TACE alone group had more TAMs with positive F4/80 staining compared with the sham TACE group (percentage of TAMs in tumor infiltrating lymphocytes, $42.2 \pm 3.7 \%$ vs. $18.5 \pm 3.2 \%$; $\mathrm{P}<0.01$ ). Combination with $\mathrm{ZA}$ treatment significantly inhibited TAM infiltration following TACE in rat HCC models. The percentage of F4/80-positive TAMs in tumor infiltrating lymphocytes was reduced by $\sim 60 \%$ compared with the TACE alone group $(15.7 \pm 2.0 \%$; $\mathrm{P}<0.01$; Fig. $2 \mathrm{~B}$ and $\mathrm{C}$ ).

ZA inhibits tumor angiogenesis following TACE. In the subsequent studies, MVD and enhanced DCE-MRI were used to evaluate tumor angiogenesis following TACE in HCC models. The data revealed that at 14 days following the procedure, the TACE alone group had significantly increased MVD in tumors compared with sham TACE group $(46.8 \pm 5.2 \%$ vs. $34.8 \pm 3.6 \%$; $\mathrm{P}<0.01)$. However, the TACE combined with ZA treatment 


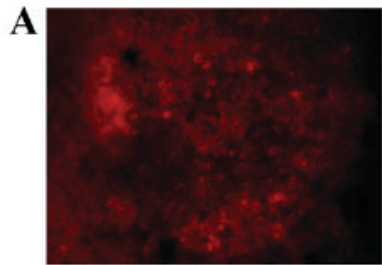

Sham TACE
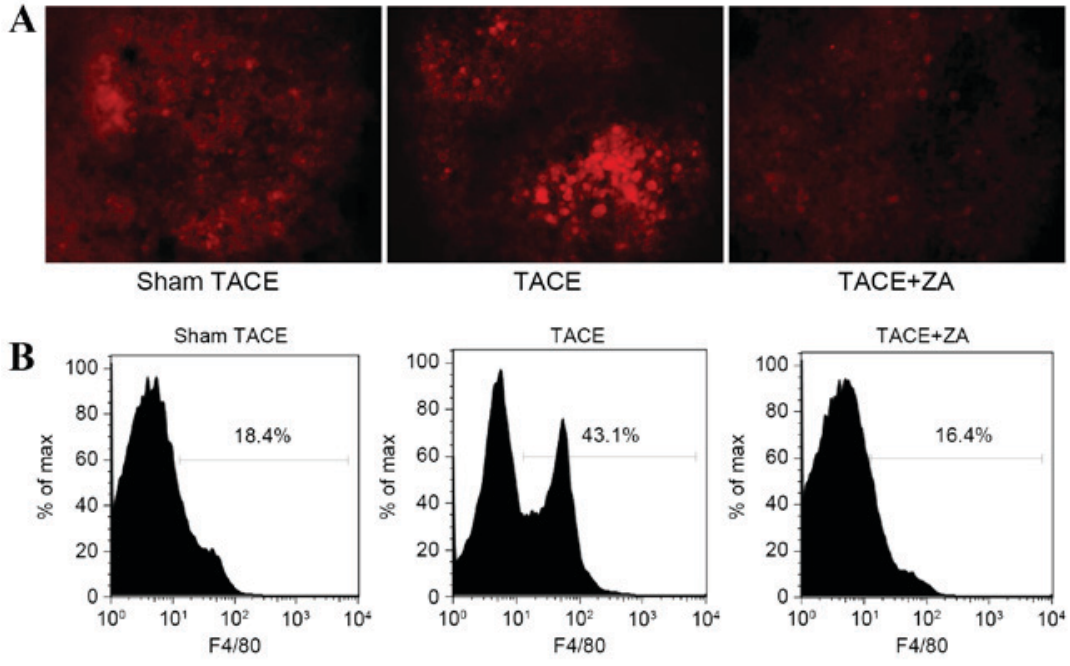

TACE+ZA
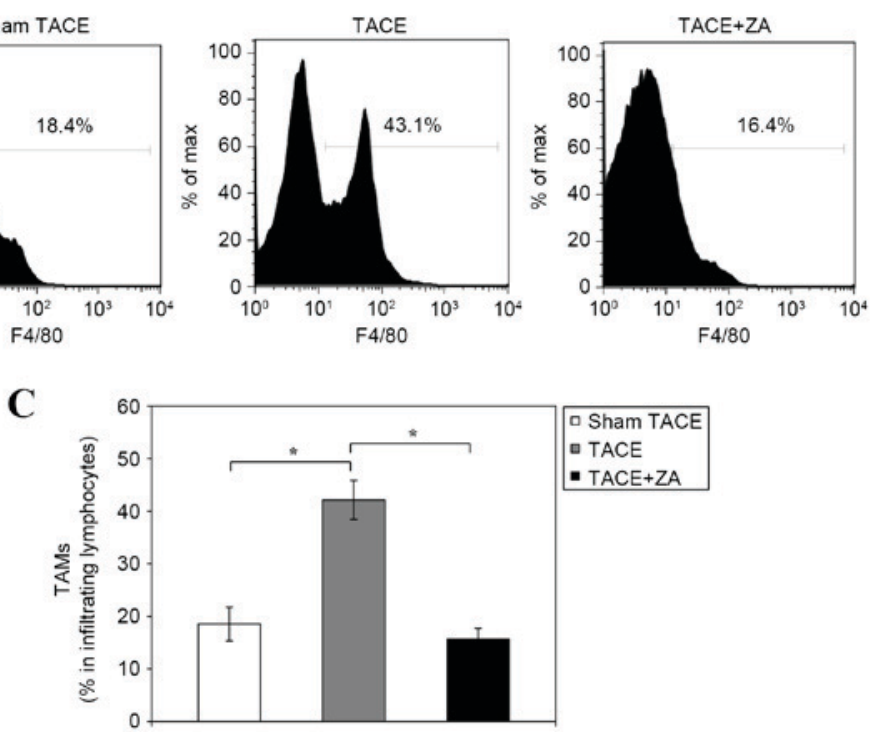

Figure 2. TAM infiltration in hepatocellular carcinoma tumors following TACE. (A) At 14 days following the procedure, TAMs in tumor tissues from sham TACE, TACE alone and TACE combined with ZA treatment groups were detected by immunohistochemical staining with anti-F4/80 antibody (magnification, $\mathrm{x} 40$ ). The figures are representative of three separate experiments. (B) Tumor infiltrating lymphocytes were isolated and stained with fluorescence conjugated anti-F4/80 antibody. Quantitative analyses were then performed using flow cytometry. The data are expressed as the percentage of F4/80-positive cells in the tumor infiltrating lymphocyte population. The figures are representative of three separate experiments. (C) Histogram of TAM percentages in tumor infiltrating lymphocytes by flow cytometry analysis. The data are expressed as the mean \pm standard deviation. ${ }^{*} \mathrm{P}<0.01$. TAMs, tumor associated macrophages. TACE, transcatheter arterial chemoembolization; ZA, zoledronic acid.

group had inhibited tumor MVD compared with TACE alone group (24.6 $\pm 1.8 \%$ vs. $46.8 \pm 5.2 ; \mathrm{P}<0.01$; Fig. $3 \mathrm{~A}$ and $\mathrm{B})$.

IS is a proportional index of tumor angiogenesis calculated from DCE-MRI images (21). No significant differences were observed between the rat models prior to treatment. At 7 days following procedure, the TACE alone group showed decreased IS compared with the sham TACE group $(0.57 \pm 0.17$ vs. $1.53 \pm 0.32 ; \mathrm{P}<0.01)$. The TACE combined with ZA treatment group showed a more decreased IS ( $40 \%$ decrease) compared with the TACE alone group $(0.32 \pm 0.11$ vs. $0.57 \pm 0.17 ; \mathrm{P}<0.05)$. At 14 days following the procedure, the TACE alone group exhibited restored IS, which was not significantly different to the sham TACE group (1.42 \pm 0.30 vs. $1.46 \pm 0.34 ; \mathrm{P}=0.805)$. However, the group combined with $\mathrm{ZA}$ treatment had decreased IS compared with the TACE alone group $(0.35 \pm 0.09$ vs. $1.42 \pm 0.30 ; \mathrm{P}<0.01)$, which stabilized at a comparative level similar to early stage (7 days) following the procedure (Fig. 3C). These data suggested that combination with ZA treatment allowed TACE to achieve sustained inhibition of tumor angiogenesis in rat HCC models.

ZA inhibits secretion of VEGF following TACE. VEGF is generally considered to be one of the most important angiogenic factors released by macrophages. In order to establish whether secretion of VEGF may be affected by
TACE combined with ZA treatment, local and systemic VEGF levels were examined in the HCC models at 14 days following the procedure. Western blot analysis of tumor tissues revealed that the TACE alone group had notably increased VEGF expression compared with the sham TACE group, but expression may be inhibited if combined with ZA treatment following procedure (Fig. 4A). In addition, ELISA analysis revealed that the TACE alone group had significantly increased concentration of VEGF in plasma compared with the sham TACE group $(158.2 \pm 16.2 \mathrm{pg} / \mathrm{ml}$ vs. $87.3 \pm 7.0 \mathrm{pg} / \mathrm{ml}$; $\mathrm{P}<0.01)$. TACE combined with $\mathrm{ZA}$ treatment group revealed inhibited VEGF expression in plasma compared with the TACE alone group (73.8 $\pm 7.5 \mathrm{pg} / \mathrm{ml}$ vs. $158.2 \pm 16.2 \mathrm{pg} / \mathrm{ml}$; $\mathrm{P}<0.01$; Fig. 4B). These data indicated that combination with ZA treatment inhibited secretion of VEGF following TACE in rat $\mathrm{HCC}$ models.

\section{Discussion}

In the clinic, TACE is recommended to patients with advanced HCC, as it is able to deprive tumor blood supply that induce severe hypoxia and tumor necrosis (5-7). However, numerous previous studies have proposed that complete tumor necrosis is seldom achieved in patients with HCC following TACE $(10,11,29,30)$. In addition, a few studies 
A

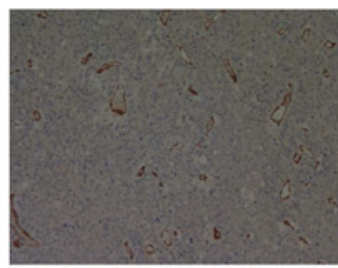

Sham TACE

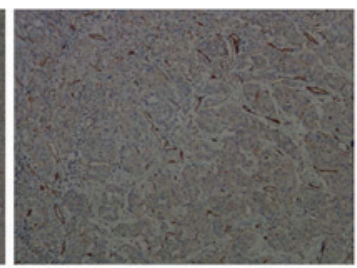

TACE

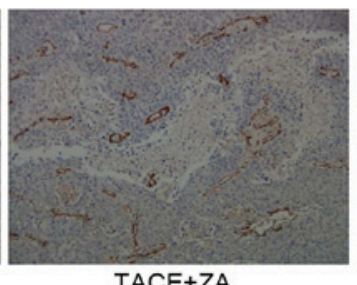

TACE+ZA
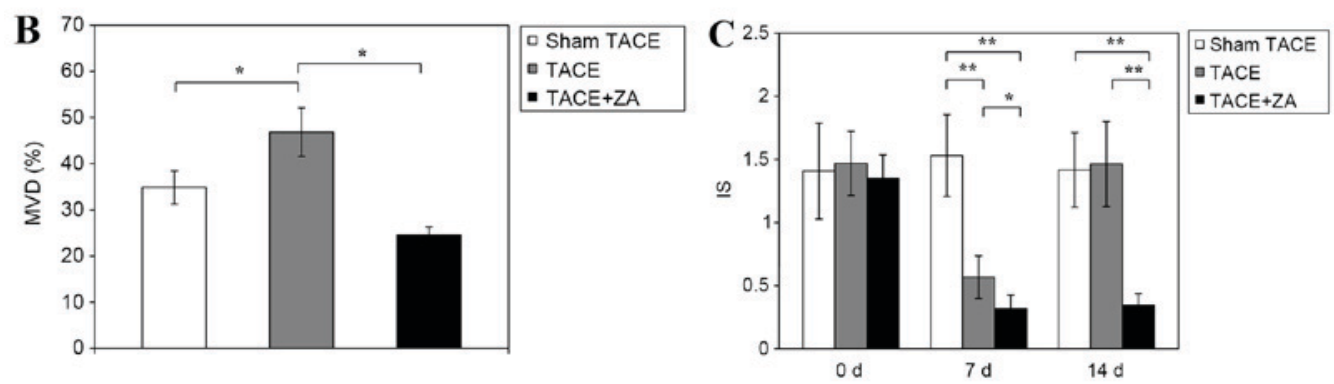

Figure 3. Tumor angiogenesis following TACE in hepatocellular carcinoma. (A) At 14 days subsequent to the procedure, vessel density of tumor tissues from various groups, including sham TACE, TACE alone and TACE combined with ZA treatment groups, was assessed by immunohistochemical staining with anti-CD31 antibody (magnification, $\mathrm{x} 40$ ). The figures are representative of three separate experiments. (B) MVD of tumor tissues was calculated by the method described in materials and methods. The data were expressed as the mean \pm standard deviation. ${ }^{*} \mathrm{P}<0.01$. (C) At 7 and 14 days following TACE, MRI was used to measure intensity-time curves of tumors with dynamic contrast enhancement. IS, a proportional index of tumor angiogenesis, was calculated from MRI images. The data are expressed as the mean \pm standard deviation. ${ }^{*} \mathrm{P}<0.05,{ }^{* * *} \mathrm{P}<0.01$. MRI, magnet resonance imaging; IS, initial slope; MVD, mean vessel density; TACE, transcatheter arterial chemoembolization; ZA, zoledronic acid.

reported biological changes in residual $\mathrm{HCC}$ following TACE $(13,14,31)$. Significantly increased VEGF expression and more angiogenesis were observed in plasma and residual tumors $(13,14,31)$. Local hypoxia occurs following establishment of a TACE-induced pro-angiogenic microenvironment, which weakens the treatment effect of the procedure and fertilizes residual tumors $(13,14,31)$. This may be one reason why complete tumor necrosis following TACE is challenging to achieve. Such a microenvironment may accelerate recurrence and metastasis of residual tumors, which leads to failure of TACE treatment. Therefore, therapeutic strategies for inhibiting tumor angiogenesis may improve outcomes of patients with $\mathrm{HCC}$ receiving with TACE treatment.

Hypoxia tumor infiltrating cells were also considered to serve an important role in modulation of microenvironment (15-17). As a primary subset of tumor infiltrating cells, TAMs have been implicated in tumor growth and prognosis in patients with $\mathrm{HCC}(22,23)$. In the present study, compared with the sham control, the TACE alone group exhibited more F4/80-positive TAMs infiltrating orthotopic tumors at 14 days following the procedure. Characteristics of the microenvironment following TACE, including local hypoxia and inflammation, evidently induced TAM infiltration.

$\mathrm{ZA}$ is established in the clinic to inhibit bone resorption, primarily in metastatic tumors $(32,33)$. It may exert an indirect effect on TAMs in certain types of tumors (34). Coscia et al (35) applied ZA with a clinically compatible dose to treat mouse mammary tumors. The results demonstrated that administration of ZA significantly reduced the number of TAMs in tumors. Using ZA in liver tumors, Zhang et al (36) found that it ZA improved the antitumor effects of sorafinib as a TAM inhibitor. The present data indicated that combination with ZA treatment enhanced the TACE-induced inhibition of

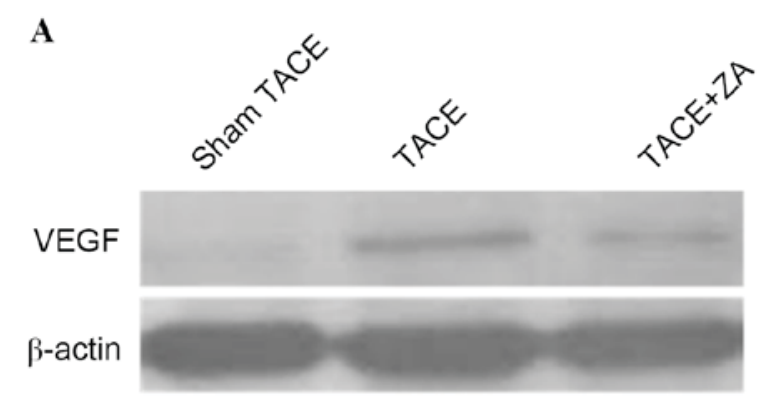

B

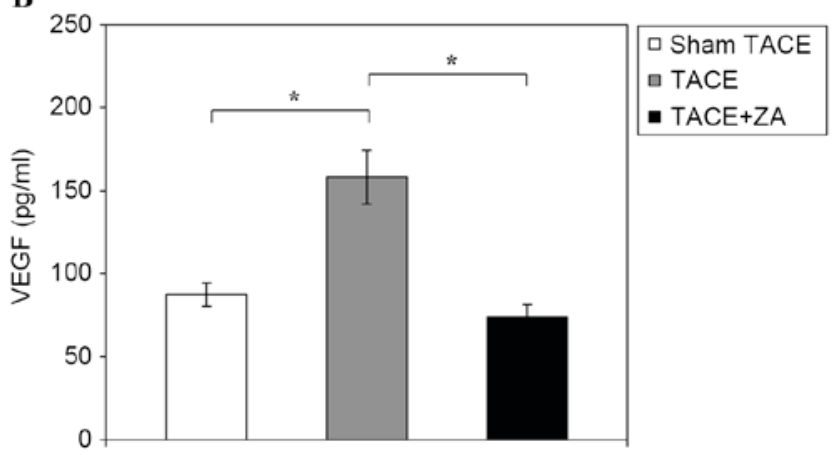

Figure 4. Secretion of VEGF following TACE in hepatocellular carcinoma. (A) At 14 days subsequent to the procedure, VEGF expression of tumor tissues from sham TACE, TACE alone and TACE combined with ZA treatment groups was analyzed by western blotting. The data are representative of three separate experiments. (B) At 14 days following the procedure, the concentration of VEGF in plasma was determined by ELISA analysis. The data are expressed as the mean \pm standard deviation. ${ }^{*} \mathrm{P}<0.01$. VEGF, vascular endothelial growth factor; TACE, transcatheter arterial chemoembolization; ZA, zoledronic acid. 
tumor growth in rat HCC models. Mechanism investigation demonstrated that ZA significantly inhibited TAM infiltration following TACE.

TAMs have been reported to facilitate angiogenesis in non-liver tumors $(21,37)$. In accordance with these findings, the present data identified that the combination with ZA treatment enabled sustained inhibition of tumor angiogenesis following TACE in rat HCC models. In addition, the present study revealed that ZA treatment inhibits secretion of VEGF following TACE. VEGF is an important pro-angiogenic factor released by TAMs, which is involved in the initiation and remodeling of tumor angiogenesis (34). ZA treatment inhibited tumor angiogenesis following TACE, which was mediated by VEGF secreted from TAMs.

In conclusion, combination with ZA treatment enhanced the effects of TACE through inhibiting TAM infiltration and tumor angiogenesis following TACE in rat HCC models. It may serve as a novel therapeutic strategy for improving the outcomes of TACE treatment in patients with advanced HCC.

\section{Acknowledgements}

The present study was supported by grants from the Scientific Research Foundation of Jiangsu Province (grant no. BK20130259) and the National Natural Science Foundation of China (grant no. 31000526).

\section{References}

1. El-Serag HB and Rudolph KL: Hepatocellular carcinoma: Epidemiology and molecular carcinogenesis. Gastroenterology 132: 2557-2576, 2007.

2. Connell LC, Harding JJ and Abou-Alfa GK: Advanced hepatocellular cancer: The current state of future research. Curr Treat Options Oncol 17: 43, 2016

3. Llovet JM, Zucman-Rossi J, Pikarsky E, Sangro B, Schwartz M, Sherman M and Gores G: Hepatocellular carcinoma. Nat Rev Dis Primers 2: 16018, 2016.

4. Han KH, Kudo M, Ye SL, Choi JY, Poon RT, Seong J, Park JW, Ichida T, Chung JW, Chow P and Cheng AL: Asian consensus workshop report: Expert consensus guideline for the management of intermediate and advanced hepatocellular carcinoma in Asia. Oncology 81 (Suppl 1): S158-S164, 2011.

5. Bruix J and Sherman M; American Association for the Study of Liver Diseases: Management of hepatocellular carcinoma: An update. Hepatology 53: 1020-1022, 2011.

6. Benson AB III, Abrams TA, Ben-Josef E, Bloomston PM, Botha JF, Clary BM, Covey A, Curley SA, D'Angelica MI, Davila R, et al: NCCN clinical practice guidelines in oncology: Hepatobiliary cancers. J Natl Compr Canc Netw 7: 350-391, 2009

7. Lo CM, Ngan H, Tso WK, Liu CL, Lam CM, Poon RT, Fan ST and Wong J: Randomized controlled trial of transarterial lipiodol chemoembolization for unresectable hepatocellular carcinoma. Hepatology 35: 1164-1171, 2002.

8. Kobayashi T, Ishiyama K and Ohdan H: Prevention of recurrence after curative treatment for hepatocellular carcinoma. Surg Today 43: 1347-1354, 2013.

9. Llovet JM, Real MI, Montaña X, Planas R, Coll S, Aponte J, Ayuso C, Sala M, Muchart J, Solà R, et al: Arterial embolisation or chemoembolisation versus symptomatic treatment in patients with unresectable hepatocellular carcinoma: A randomised controlled trial. Lancet 359: 1734-1739, 2002.

10. Matsuo N, Uchida H, Nishimine K, Soda S, Oshima M, Nakano H, Nagano N, Nishimura Y, Yoshioka T, Guo Q, et al: Segmental transcatheter hepatic artery chemoembolization with iodized oil for hepatocellular carcinoma: Antitumor effect and influence on normal tissue. J Vasc Interv Radiol 4: 543-549, 1993.

11. Higuchi T, Kikuchi M and Okazaki M: Hepatocellular carcinoma after transcatheter hepatic arterial embolization. A histopathologic study of 84 resected cases. Cancer 73: 2259-2267, 1994.
12. Weintraub JL and Salem R: Treatment of hepatocellular carcinoma combining sorafenib and transarterial locoregional therapy: State of the science. J Vasc Interv Radiol 24: 1123-1134, 2013.

13. Wang B, Xu H, Gao ZQ, Ning HF, Sun YQ and Cao GW: Increased expression of vascular endothelial growth factor in hepatocellular carcinoma after transcatheter arterial chemoembolization. Acta Radiol 49: 523-529, 2008.

14. Sergio A, Cristofori C, Cardin R, Pivetta G, Ragazzi R, Baldan A, Girardi L, Cillo U, Burra P, Giacomin A and Farinati F: Transcatheter arterial chemoembolization (TACE) in hepatocellular carcinoma (HCC): The role of angiogenesis and invasiveness. Am J Gastroenterol 103: 914-921, 2008.

15. Mantovani A, Allavena P, Sica A and Balkwill F: Cancer-related inflammation. Nature 454: 436-444, 2008.

16. Mantovani A, Garlanda C and Allavena P: Molecular pathways and targets in cancer-related inflammation. Ann Med 42: 161-170, 2010.

17. Friedl $\mathrm{P}$ and Alexander S: Cancer invasion and the microenvironment: Plasticity and reciprocity. Cell 147: 992-1009, 2011.

18. Capece D, Fischietti M, Verzella D, Gaggiano A, Cicciarelli G, Tessitore A, Zazzeroni F and Alesse E: The inflammatory microenvironment in hepatocellular carcinoma: A pivotal role for tumor-associated macrophages. Biomed Res Int 2013: 187204, 2013.

19. Riabov V, Gudima A, Wang N, Mickley A, Orekhov A and Kzhyshkowska J: Role of tumor associated macrophages in tumor angiogenesis and lymphangiogenesis. Front Physiol 5: 75, 2014.

20. Bingle L, Brown NJ and Lewis CE: The role of tumour-associated macrophages in tumour progression: Implications for new anticancer therapies. J Pathol 196: 254-265, 2002.

21. Leek RD and Harris AL: Tumor-associated macrophages in breast cancer. J Mammary Gland Biol Neoplasia 7: 177-189, 2002.

22. Ding T, Xu J, Wang F, Shi M, Zhang Y, Li SP and Zheng L: High tumor-infiltrating macrophage density predicts poor prognosis in patients with primary hepatocellular carcinoma after resection. Hum Pathol 40: 381-389, 2009.

23. Zhu XD, Zhang JB, Zhuang PY, Zhu HG, Zhang W, Xiong YQ, Wu WZ, Wang L, Tang ZY and Sun HC: High expression of macrophage colony-stimulating factor in peritumoral liver tissue is associated with poor survival after curative resection of hepatocellular carcinoma. J Clin Oncol 26: 2707-2716, 2008.

24. Chanmee T, Ontong P, Konno K and Itano N: Tumor-associated macrophages as major players in the tumor microenvironment. Cancers (Basel) 6: 1670-1690, 2014.

25. Gupta S, Kobayashi S, Phongkitkarun S, Broemeling LD and Kan Z: Effect of transcatheter hepatic arterial embolization on angiogenesis in an animal model. Invest Radiol 41: 516-521, 2006.

26. Weidner N: Current pathologic methods for measuring intratumoral microvessel density within breast carcinoma and other solid tumors. Breast Cancer Res Treat 36: 169-180, 1995.

27. Thoeny HC, De Keyzer F, Vandecaveye V, Chen F, Sun X, Bosmans H, Hermans R, Verbeken EK, Boesch C, Marchal G, et al: Effect of vascular targeting agent in rat tumor model: Dynamic contrast-enhanced versus diffusion-weighted MR imaging. Radiology 237: 492-499, 2005.

28. Buadu LD, Murakami J, Murayama S, Hashiguchi N, Sakai S, Masuda K, Toyoshima S, Kuroki S and Ohno S: Breast lesions: Correlation of contrast medium enhancement patterns on MR images with histopathologic findings and tumor angiogenesis. Radiology 200: 639-649, 1996.

29. Bharat A, Brown DB, Crippin JS, Gould JE, Lowell JA, Shenoy S, Desai NM and Chapman WC: Pre-liver transplantation locoregional adjuvant therapy for hepatocellular carcinoma as a strategy to improve longterm survival. J Am Coll Surg 203: 411-420, 2006

30. Duvoux C, Cherqui D, Van Nhieu JT, Vavasseur D, Zafrani ES, Mathieu D, Fagniez PL and Dhumeaux D: Chemoembolization for hepatocellular carcinoma in cirrhotic patients: Assessment of efficacy on total hepatectomy specimens. Transplant Proc 26: 3572-3573, 1994

31. Liao X, Yi J, Li X, Yang Z, Deng W and Tian G: Expression of angiogenic factors in hepatocellular carcinoma after transcatheter arterial chemoembolization. J Huazhong Univ Sci Technolog Med Sci 23: 280-282, 2003.

32. Zekri J, Mansour M and Karim SM: The anti-tumour effects of zoledronic acid. J Bone Oncol 3: 25-35, 2014. 
33. Green J and Lipton A: Anticancer properties of zoledronic acid. Cancer Invest 28: 944-957, 2010.

34. Rogers TL and Holen I: Tumour macrophages as potential targets of bisphosphonates. J Transl Med 9: 177, 2011.

35. Coscia M, Quaglino E, Iezzi M, Curcio C, Pantaleoni F, Riganti C, Holen I, Mönkkönen H, Boccadoro M, Forni G, et al: Zoledronic acid repolarizes tumour-associated macrophages and inhibits mammary carcinogenesis by targeting the mevalonate pathway. J Cell Mol Med 14: 2803-2815, 2010.
36. Zhang W, Zhu XD, Sun HC, Xiong YQ, Zhuang PY, Xu HX, Kong LQ, Wang L, Wu WZ and Tang ZY: Depletion of tumor-associated macrophages enhances the effect of sorafenib in metastatic liver cancer models by antimetastatic and antiangiogenic effects. Clin Cancer Res 16: 3420-3430, 2010.

37. Condeelis J and Pollard JW: Macrophages: Obligate partners for tumor cell migration, invasion, and metastasis. Cell 124: 263-266, 2006 